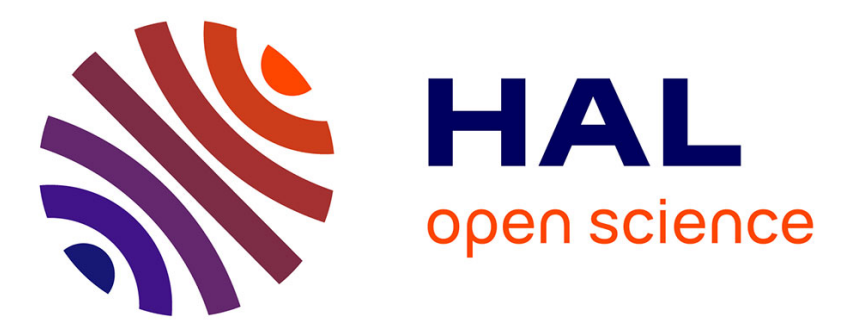

\title{
An MCMC algorithm for BOC and AltBOC signaling acquisition in multipath environments
}

\author{
Fadoua Brahim, Thierry Chonavel, Olivier Rabaste
}

\section{To cite this version:}

Fadoua Brahim, Thierry Chonavel, Olivier Rabaste. An MCMC algorithm for BOC and AltBOC signaling acquisition in multipath environments. IEEE/ION PLANS 2008: position location and navigation symposium, may 5-8, Monterey, California, USA, May 2008, Monterey, United States. pp.424 - 432. hal-02134554

\section{HAL Id: hal-02134554 \\ https://hal.science/hal-02134554}

Submitted on 27 May 2019

HAL is a multi-disciplinary open access archive for the deposit and dissemination of scientific research documents, whether they are published or not. The documents may come from teaching and research institutions in France or abroad, or from public or private research centers.
L'archive ouverte pluridisciplinaire HAL, est destinée au dépôt et à la diffusion de documents scientifiques de niveau recherche, publiés ou non, émanant des établissements d'enseignement et de recherche français ou étrangers, des laboratoires publics ou privés. 


\section{An MCMC Algorithm for BOC and AltBOC Signaling Acquisition in Multipath Environments}

\author{
Fadoua Brahim and Thierry Chonavel \\ TELECOM-Bretagne \\ Signal and Communication department \\ Technopole Brest Iroise \\ Brest, France 29238 \\ Email: fadoua.brahim@telecom-bretagne.eu
}

\author{
Olivier Rabaste \\ Laboratoire AstroParticule et Cosmologie \\ 10 rue Alice Domon et Lonie Duquet \\ 75205 Paris Cedex 13, France
}

\begin{abstract}
The Binary Offset Carrier (BOC) and Alternate BOC (AltBOC) waveforms, introduced for Galileo signaling, are designed to enable high time delay estimation accuracy. However, their autocorrelation functions have multiple narrow peaks, thus leading to potential acquisition bias, in particular in presence of multipaths. In order to detect the first path, a high sampling rate is considered. Unfortunately, this introduces noise correlation. In this paper, we compare a technique developed for multipath channel estimation based on Monte Carlo Markov Chain (MCMC) simulation with the standard acquisition approach, in multipath environments, considering both accuracy and computational load issues.
\end{abstract}

\section{INTRODUCTION}

Multipath error is the main source of error in high accuracy navigation, often leading to an estimation bias of tens of meters [13].

There are two stages for the generation of a synchronized $\mathrm{PN}$-code at the receiver side: acquisition (or coarse estimation) followed by tracking (or fine estimation). In order to deal with multipath errors, we need to narrow the spacing $\Delta$ between early and late correlators at the tracking stage. However, the Early-Late tracking loop hold range is about $[-\Delta, \Delta]$. Thus, the acquisition stage should give an estimation of the delay within this range. The more severe the multipaths are, the narrower the hold range should be, and the more precise the acquisition should be. In this paper, we deal with the acquisition precision problem, and propose an algorithm which is more accurate than the classical one. In fact, the acquisition traditional approach commonly used in GPS receivers is not accurate enough when BOC and AltBOC modulations are used. Actually, the autocorrelation function of such modulations presents a sharp main peak that enables highly accurate code tracking [1] and good multipath resolution, but it also presents many side lobes that lead to acquisition ambiguities [18].

According to the traditional approach, the receiver sequentially scans all possible combinations of frequency/code in the search space, until the correlation value exceeds a certain predefined threshold. However, the autocorrelation function is distorted by close paths. Then, we cannot distinguish the first path among their contributions, thus leading to a biased acquisition.
In fact, during its propagation to a receiver, the transmitted signal is diffracted and reflected off surrounding objects, including the earth's surface [17]. Reflections lead to the presence of multipaths at the receiver side, thet is several correlation peaks are measured while diffractions lead to corrupted correlation waveform of the received signal (theoretically symmetric). Both types of degradation lead to incorrect measurements of the pseudo-distance when using the classical acquisition. Thus, the classical acquisition method will not achieve expected accuracy.

The goal of this paper is to promote multipath identification for use in BOC and AltBOC modulated signal receivers to eliminate multipath errors. In the literature, several methods have been proposed to estimate multiple possibly overlapping components of the same signal buried in additive noise such as Matching Pursuit [4] or EM [5]. These approaches consider a parametric model of the multipath channel. In a bayesian framework, it has also been proposed to model the multipath channel via a prior Bernoulli-Gaussian distribution and solve the path detection and estimation problem by means of MCMC methods [12]. This kind of techniques has recently been applied to numerous different applications, i.e. geophysics [19], astrophysics [2] and underwater acoustics [16]. Its main advantage is its capability to recover each contribution of interfering paths. In [16], algorithms have been adapted to cope with noise correlation at the output of the matched filtering. However, it is well known that the MCMC method is costly when applied to large data sets and applying it to a minimum Galileo spreading sequence of 4092 chips would be very time consuming.

Our method involves using first the traditional approach to determine a reduced zone where the matched filter output energy is maximum, and then applying the MCMC algorithm over this reduced zone. Then, the main autocorrelation function peak can be recovered, and the calculation burden remains quite acceptable.

This paper is organized as follows. First, we introduce the autocorrelation features of $\mathrm{BOC}$ and AltBOC modulated signals used in Galileo. Next, we present the classical scheme of acquisition and introduce our method. Finally, the main performance characteristics of these algorithms are discussed 
and compared.

\section{SignALING FEATURES}

A direct sequence spread spectrum signal an be represented as follows:

$$
s(t)=\sum_{n=-\infty}^{\infty} a_{n} q\left(t-n T_{c}\right),
$$

where the $\left\{a_{k}\right\}$ represent binary values $(+1$ or -1$)$ of the spreading sequence, while $q(t)$ is the spreading waveform, and $T_{c}$ is the spreading code period.

\section{A. $B O C(m, n)$ structure}

For the BOC modulations considered in this paper, the spreading symbol is divided into $\mathrm{K}$ segments. Then, the spreading symbol is given by:

$$
q(t)=\sum_{k=0}^{K-1} c_{k} p_{T_{c} / K}\left(t-k T_{c} / K\right),
$$

where

$$
p_{\Delta}(t)= \begin{cases}1, & 0<t<\Delta \\ 0, & \text { elsewhere }\end{cases}
$$

and $\left\{c_{0}, c_{1}, \ldots, c_{K-1}\right\} \in \pm 1, T_{c}$ is the chip duration, and $T_{c} / K$ is the segment duration also called the subcarrier duration.

A sine-phased BOC modulation with subcarrier frequency $f_{s} \times 1,023 \mathrm{MHz}$ and spreading code rate $f_{c} \times 1,023 \mathrm{MHz}$, also denoted $\operatorname{BOCsin}\left(f_{s}, f_{c}\right)$, uses the sequence $[1,-1,1, \ldots]$ where the number of transitions from 1 to -1 or -1 to 1 is equal to $K=2 f_{s} / f_{c}-1$, an integer that may be either even or odd.

A cosine-phased BOC modulation with subcarrier frequency $f_{s} \times 1,023 \mathrm{MHz}$ and spreading code rate $f_{c} \times$ $1,023 \mathrm{MHz}$, also denoted $\mathrm{BOC} \cos \left(f_{s}, f_{c}\right)$, uses the sequence $[1,-1,-1,1,1,-1,-1, \ldots]$ where the number of transitions from 1 to -1 or -1 to 1 is equal to $K=2 f_{s} / f_{c}-1$, an integer that may be either even or odd.

Clearly sine and cosine BOC sequences can be seen as quantized sine or cosine waves at frequency $f_{s}$.

There, we are more specifically interested in open service signals. Thus, we focus on the $\mathrm{BOC} \sin (1,1)$ modulation used in E1 signal. In fact, BOCsin $(1,1)$ is the modulation used in the Galileo E1 signal with a carrier frequency of $1575.42 \mathrm{MHz}$, a code length of 4092 chips and code duration of $4 \mathrm{~ms}$.

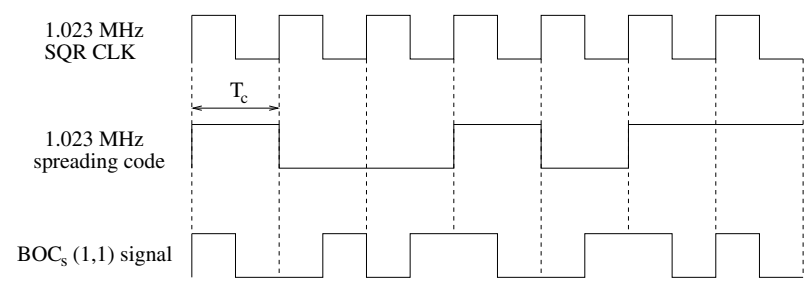

Fig. 1. Example 1 : BOCsin(1,1) (number of transitions: $K=2 *$ $\frac{1}{1}-1=1$ )

\section{B. AltBOC structure}

The AltBOC signal is defined as the product of a PRN code sequence with a complex sub-carrier. In the E5 open service signal used in Galileo, the AltBOC can be expressed as

$$
\begin{aligned}
x_{A l t B O C}(t)= & \left(c_{L}+j \cdot c_{L}^{\prime}\right) \cdot\left[s c_{a s}(t)-j \cdot s c_{a s}\left(t-\frac{T_{s}}{4}\right)\right] \\
+ & \left(c_{U}+j \cdot c_{U}^{\prime}\right) \cdot\left[s c_{a s}(t)+j \cdot s c_{a s}\left(t-\frac{T_{s}}{4}\right)\right] \\
+ & \left(\overline{c_{L}}+j \cdot \overline{c_{L}^{\prime}}\right) \cdot\left[s c_{a p}(t)-j \cdot s c_{a p}\left(t-\frac{T_{s}}{4}\right)\right] \\
+ & \left(\overline{c_{U}}+j \cdot \overline{c_{U}^{\prime}}\right) \cdot\left[s c_{a p}(t)+j \cdot s c_{a p}\left(t-\frac{T_{s}}{4}\right)\right],
\end{aligned}
$$

where $\overline{c_{L}}=c_{U} c_{U}^{\prime} c_{L}^{\prime}, \overline{c_{L}^{\prime}}=c_{U} c_{U}^{\prime} c_{L}, \overline{c_{U}}=c_{L} c_{U}^{\prime} c_{L}^{\prime}$ and $\overline{c_{U}^{\prime}}=c_{U} c_{L} c_{L}^{\prime}$ and $c_{U}$ being the upper data code commonly known as E5bI, $c_{U}^{\prime}$ the pilot upper code known as E5bQ, $c_{L}$ the data lower code known as E5aI and $c_{L}^{\prime}$ the pilot lower code known as E5aQ.

$$
\begin{array}{rlr}
s c_{a s}(t) & = & \frac{\sqrt{2}}{4} \operatorname{sign}\left(\cos \left(2 \pi f_{s} t-\frac{\pi}{4}\right)\right) \\
+ & \frac{1}{2} \operatorname{sign}\left(\cos \left(2 \pi f_{s} t\right)\right) \\
+ & \frac{\sqrt{2}}{4} \operatorname{sign}\left(\cos \left(2 \pi f_{s} t+\frac{\pi}{4}\right)\right)
\end{array}
$$

and

$$
\begin{aligned}
& s c_{a p}(t)=\quad-\frac{\sqrt{2}}{4} \operatorname{sign}\left(\cos \left(2 \pi f_{s} t-\frac{\pi}{4}\right)\right) \\
& +\quad \frac{1}{2} \operatorname{sign}\left(\cos \left(2 \pi f_{s} t\right)\right) \\
& +\quad \frac{\sqrt{2}}{4} \operatorname{sign}\left(\cos \left(2 \pi f_{s} t+\frac{\pi}{4}\right)\right) \text {. }
\end{aligned}
$$

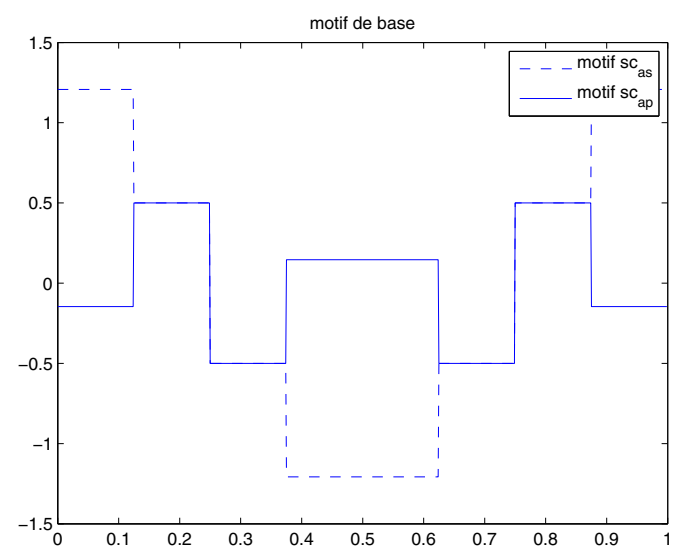

Fig. 2. Shapes of sub-carriers $s c_{a s}$ and $s c_{a p}$. 
Particularly in the Galileo context, the $\operatorname{AltBOC}(15,10)$ is used in the E5 band with a carrier frequency of 1191.795 $\mathrm{MHz}$ and a chip rate of $10.23 \mathrm{Mcps}$.

\section{Autocorrelation functions}

BOC waveforms have autocorrelation functions containing multiple peaks. There are $m / n$ sidelobes on each side of the main peak.

It comes from Eq. (1) that this signal has the following autocorrelation function:

$$
E\left[s(t) s^{*}(t-\tau)\right]=\frac{1}{T_{c}} \sum_{m} R_{a}(m) \times R_{q}\left(\tau-m T_{c}\right)
$$

where $R_{a}$ and $R_{q}$ are autocorrelations of sequence $\left\{a_{n}\right\}$ and signal $q(t)$ respectively. The theoretical autocorrelation of a BOCsin(n,n) signal possesses three peaks in the interval $\left[-T_{c},+T_{c}\right]$.

More precisely, in this case,

$$
\begin{aligned}
& R_{s}(\tau)=\operatorname{tri}(\tau)-\frac{1}{2}\left(\operatorname{tri}\left(\frac{\tau-\frac{1}{2}}{\frac{1}{2}}\right)+\operatorname{tri}\left(\frac{\tau+\frac{1}{2}}{\frac{1}{2}}\right)\right) \\
& \text { for } \quad x \leq 1 \mathrm{chip}
\end{aligned}
$$

where $x \longrightarrow \operatorname{tri}(x / y)$ is the triangle function of width $2 y$, centered at $x=0$ with $\operatorname{tri}(0)=1$.

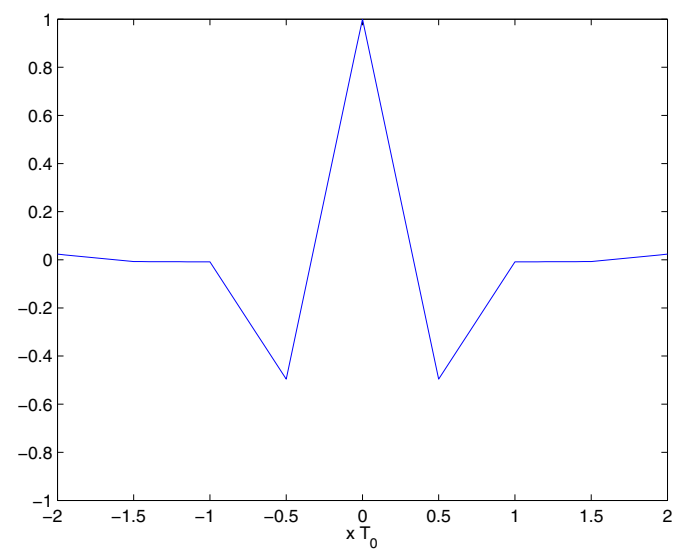

Fig. 3. BOCsin $(1,1)$ autocorrelation function

As we are interested in Open Service signals, the BOC$\sin (1,1)$ modulation that is used in the E1 Galileo signal will be considered hereafter.

The AltBOC autocorrelation function is given by [18]:

$$
\begin{aligned}
R_{x_{\text {AltBOC}}(\tau)=} & R_{c_{U}}(\tau) \times R_{a}(\tau)+R_{c_{U}} \times R_{b}(\tau) \\
+ & R_{c_{U}^{\prime}}(\tau) \times R_{a}(\tau)+R_{c_{U}^{\prime}} \times R_{b}(\tau) \\
+ & R_{c_{L}}(\tau) \times R_{a}(\tau)+R_{c_{L}} \times R_{b}(\tau) \\
+ & R_{c_{L}^{\prime}}(\tau) \times R_{a}(\tau)+R_{c_{L}^{\prime}} \times R_{b}(\tau)
\end{aligned}
$$

assuming that the crosscorrelation between the different codes is equal to zero.
The autocorrelation function also presents side peaks and the main peak width is clearly very small.

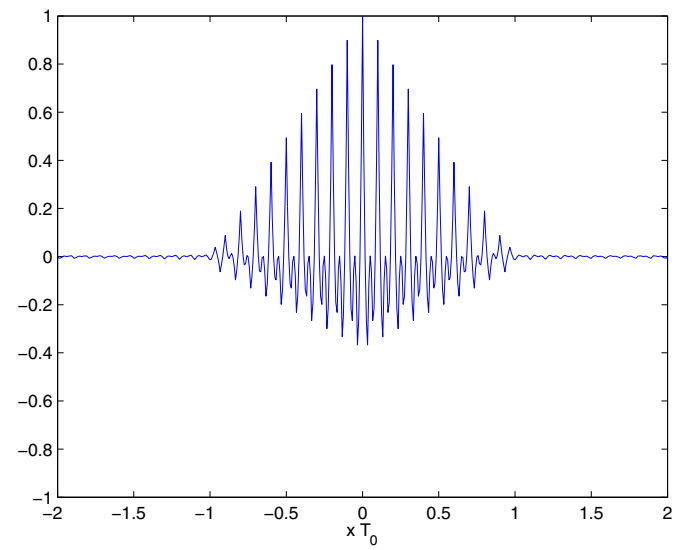

Fig. 4. AltBOC $(15,10)$ autocorrelation function

In Figure 4, the $\operatorname{AltBOC}(15,10)$ autocorrelation function is shown. This signal is used in the E5 band for Open Service use.

\section{MULTIPATH PROPAGATION}

Multipaths are mainly caused by reflection, diffraction and scattering which are the three basic mechanisms of radio propagation [17]. All three phenomena cause radio signal distortions and give rise to signal fades, as well as additional signal propagation losses. This is because they create additional radio propagation paths beyond the direct optical LOS (line of sight) path between the transmitter and the receiver, resulting in multipath fading.

The multipaths induced signal attenuation is mainly driven by the number, strength and delay of the multipath signals. The signal components arriving from indirect paths (which are delayed and attenuated versions of the original signal) and the direct LOS path (if it exists), combine and produce a distorted version of the transmitted signal. The combination may cause constructive and/or destructive interference effects [11]. Many studies aim at characterizing the different kinds of GPS channels, especially the indoor [7], [14] and the urban [15], [20] channels. The studies were conducted using either simulated models [21] and [6] or field tests [15], [20].

In this paper, we tested an MCMC approach, namely MPM (maximum posterior mode) method on the two types of channels described below.

\section{A. Simulated channel}

In order to show the high accuracy of our method, we chose a channel such that the path separations are less than 1 chip. We considered a number of paths that follows a Poisson distribution. As shown in Figure 5, the first path is considered at the beginning of the chip and its amplitude is taken with a Rice distribution, whereas the other paths are uniformly distributed on 1 chip and their amplitudes are distributed according to a Rayleigh distribution. 


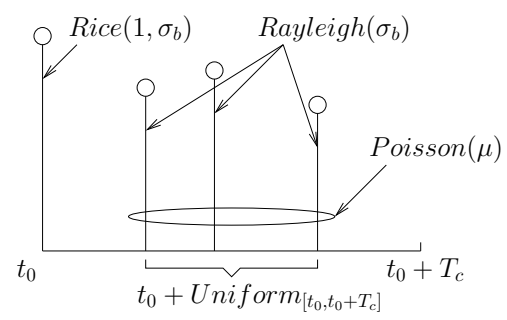

Fig. 5. An example of a simulated channel

\section{B. Real channel}

Following Steingass [20] [21], a statistical analysis of the navigation multipath channel is achieved through several measurements. In fact, the channel was measured from a simulated satellite (a zeppelin) to a receiver in critical urban, suburban and rural scenarios. From the received data, a channel model was derived to approximately synthesize the measured impulse response. This model allows the realistic simulation of the multipath channel by approximating every single reflection. We carried out simulations using this model to show the high performance of the MCMC method.

\section{NOTATIONS}

Considering Doppler effects, we assume the time-varying channel impulse response

$$
h_{t}(\tau)=\sum_{p=1}^{P} \alpha_{p} e^{j 2 \pi f_{d_{p}}\left(t-\tau_{p}\right)} \delta\left(\tau-\tau_{p}\right)
$$

where $P$ is the number of paths, $\alpha_{p}$ is the path amplitude, $f_{d_{p}}$ is the Doppler shift and $\tau_{p}$ is the path delay. The received signal is:

$$
\begin{aligned}
x(t) & =\left(h_{t} * s\right)(t)+n(t) \\
& =\sum_{p=1}^{P} \alpha_{p} e^{j 2 \pi f_{d_{p}}\left(t-\tau_{p}\right)} s\left(t-\tau_{p}\right)+n(t)
\end{aligned}
$$

where $n(t)$ is the correlated additive Gaussian noise. After demodulation and matched filtering at a particular frequency $f_{k}$, the signal can be written as:

$$
x\left(f_{k}, t\right)=x(t) e^{-j 2 \pi f_{k} t} * s^{*}(-t)
$$

where $*$ represents the convolution and $\lambda(t)$ is the autocorrelation function of the transmitted sequence $s(t)$.

\section{A. Ambiguity function}

When unknown Doppler shifts occur on the channel paths, one may want to apply matched filtering for different frequencies. A widely used transformation that is of interest in this case is the ambiguity function, defined as

$$
A_{s}(\tau, f)=\int_{-\infty}^{+\infty} s(t) s^{*}(t-\tau) e^{-j 2 \pi f t} d t
$$

Indeed, for a fixed $f$, the function $\tau \mapsto A_{s}(\tau, f)$ represents the output of a matched filter when the input signal has been Doppler-shifted by $f$. A simple way to build a rough version of the ambiguity function is to use a filter bank composed of matched filters spanning a certain frequency interval $\left[-f_{\max }, f_{\max }\right]$, where $f_{\max }$ is the maximum Doppler shift. As shown in Figure 6, the ambiguity function of the GPS C/A signal is characterized by a peak at the searched time delay and frequency shift. This characteristic enables the search of multipath in the time-frequency space by analysing the content of the observation at the output of the filter bank. That is to say, to decouple the estimation of time delay from the Doppler shift. That's why it is possible to apply the MCMC algorithm to each filter bank output in order to estimate the time delays.

Actually, the received signal after demodulation and matched filtering at a particular frequency $f_{k}$ is simply the convolution between $h(t)$ and the autocorrelation function $\lambda(t)$ of the transmitted sequence $s(t)$ plus a correlated additive noise. From Eq.11 and Eq.12, we get

$$
x(f, \tau)=\sum_{p=1}^{P} \alpha_{p} A_{s}\left(\tau-\tau_{p}, f-f_{p}\right)+w(f, \tau)
$$

For each analysed frequency $f_{k}\left(f_{k} \in\left[-f_{\max },+f_{\max }\right]\right)$, $x(f, \tau)$ is sampled, leading to a vector representation of the form

$$
\mathbf{x}=\mathbf{S}_{\lambda} \mathbf{h}+\mathbf{n}
$$

where $S_{\lambda}$ is the convolution matrix associated with $\lambda$ and $\mathbf{x}$ and $\mathbf{n}$ are sampled versions of $x(t)$ and $n(t)$. Note that for signals under consideration in geopositionning at each frequency $f_{k}$ function $\tau \longrightarrow A_{s}(f, \tau)$ is about the same, up to some attenuation coefficient coming from the value of the ambiguity function at the frequency $\mathrm{f}$. Thus, $h_{f}$ is a vector with $\mathrm{P}$ non zero entries with amplitudes $\alpha_{p}$

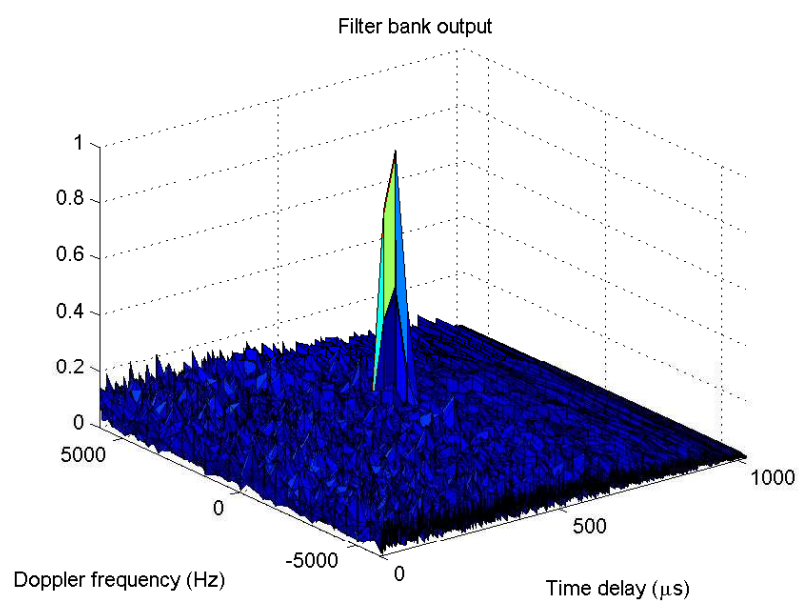

Fig. 6. Ambiguity function modulus of a GPS C/A signal in presence of one path

\section{B. The search zone}

We suggest performing a classical detection to search for the maximum energy zones. To perform detection, we 
compare the output of the correlator evaluated on each Doppler offset and each code delay, with a threshold.

The signal component with the earliest value of $t$ would represent the direct line of sight signal, (assuming that the direct line of sight to the satellite is not obstructed). Therefore, we keep only the first zone in terms of code delay. For example in Figure 7, the first zone exceeding the threshold is found between $t_{0}$ and $t_{1}$; it will therefore be our search zone, and any other ones exceeding the threshold afterwards will be discarded. This zone will be our research field where we can apply other methods of detection.

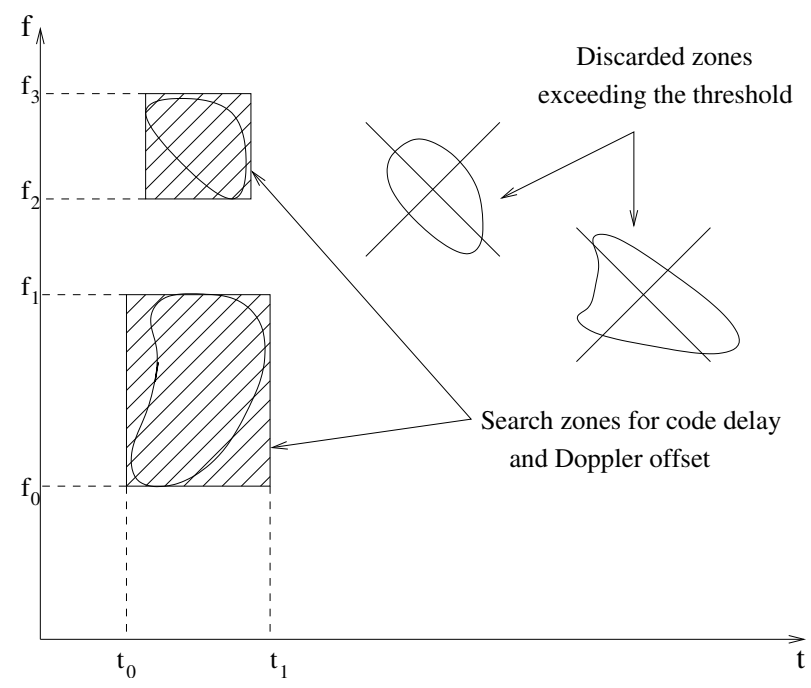

Fig. 7. Search zone for code delay and Doppler offset

\section{ClassicAl ACQUiSITION}

As defined in [8], the classical signal acquisition is a two-dimensional search process in which replica codes and carriers are correlated with the received signal. When both the code and carrier Doppler of the replica signal match the incident signal, the signal is despread and the carrier frequency is recovered. The correct alignment is identified by the maximum output power of the correlators.

The classical acquisition scheme is presented in Figure 8.

\section{A. The search space}

The search space must cover the full range of uncertainty in the code and Doppler offset range.

The Doppler range is governed by the vehicle and satellite dynamics and the stability of the receiver oscillator. The frequency resolution is determined by the coherent integration time $T$ (or dwell time). Indeed, the frequency bin width $D$ must verify $D<1 / T$ where $T$ is the integration time, since the main lobe of the signal ambiguity function is of width $1 / T$.

The code range is governed by the code length. The code resolution is a trade-off between the desired accuracy and the

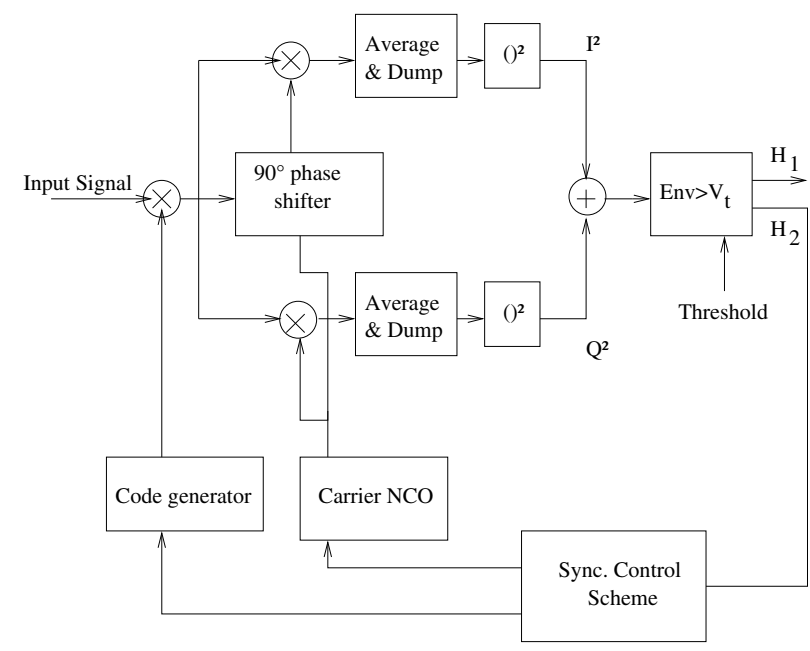

Fig. 8. Acquisition scheme

search space range. In fact, for a good resolution, the search space is too large.

Each pair of the code delay-Doppler offset values defines a "cell". Commonly, for GPS receivers, the search granularities in the code and frequency dimensions used are 0.5 code chips and $500 \mathrm{~Hz}$ respectively, as shown in Figure 9.

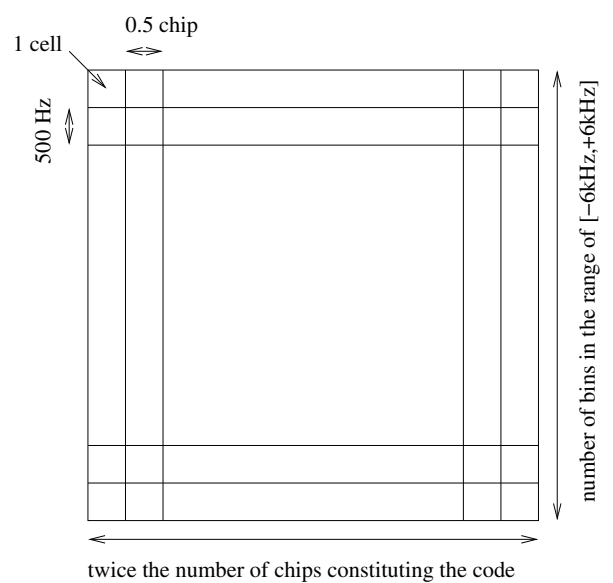

Fig. 9. Typical two dimensional acquisition search

\section{B. Detection criteria}

For each given code delay and each given Doppler offset, the correlators provide a measure of the total I (In-phase correlator output) and Q (Quadra-phase correlator output) signal voltages over the coherent integration time. The total amplitude is then given by the envelope $\sqrt{I^{2}+Q^{2}}$. This envelope is compared with a threshold $V_{t}$.

$$
\begin{aligned}
& \text { If } \sqrt{I^{2}+Q^{2}} \geq V_{t}, \quad \text { the signal is present } \\
& \text { If } \sqrt{I^{2}+Q^{2}}<V_{t}, \quad \text { the signal is absent }
\end{aligned}
$$

Following the Neyman-Pearson criterion (see Figure (10)), the threshold value can be obtained from a given false alarm 
probability. A signal is assumed to be present whenever the amplitude in a given cell is above a corresponding threshold $V_{t}$.

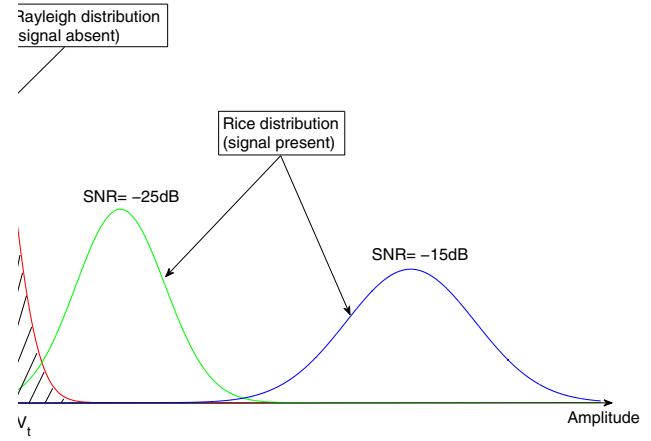

Fig. 10. Probability density functions under noise alone and noise + signal hypotheses: the gray zone defines the false alarm probability

\section{MCMC METHOD}

In order to refine detection, in each search zone we perform frequency by frequency path estimations. Each path appears at several frequencies du to signaling ambiguity function preading in the frequency domain. For the sale of simplicity, we retain the first path estimate obtained at frequency offset for which maximum amplitude path contribution is achieved.

\section{A. Bernoulli-Gaussian Model for the channel}

In order to represent the channel sparseness, we indicate two states; $q_{k}=0$ meaning the absence of a path at time $\mathrm{k}$ and $q_{k}=1$ meaning the presence of a path. Let $\mu=P\left(q_{k}=1\right)$ be the probability that a path is present at sample $k$. Then, as in [16], we define a $B G$ sequence $\mathbf{z}=(\mathbf{q}, \mathbf{h})$ where $\mathbf{h}$ is a vector of the corresponding path amplitudes to q. We model the entries $h_{k}$ of $\mathbf{h}$ by a mixture of two Gaussian distributions, such that the probability density function of $h_{k}$ conditional to $q_{k}$ is

$$
p\left(h_{k} \mid q_{k}=i\right) \sim N\left(0, \sigma_{i}^{2}\right)+j N\left(0, \sigma_{i}^{2}\right) \quad i=0,1
$$

where $\sigma_{0}$ is chosen close to zero and much smaller than $\sigma_{1}$. The estimation of $\mathbf{z}$ is achieved through the maximization of the posterior likelihood, denoted by $p(\mathbf{z} \mid \mathbf{x})$. From Bayes formula,

$$
p(\mathbf{z} \mid \mathbf{x}) \propto p(\mathbf{x} \mid \mathbf{z}) p(\mathbf{z})=p(\mathbf{x} \mid \mathbf{z}) p(\mathbf{h} \mid \mathbf{q}) p(\mathbf{q})
$$

Expressions of $p(x \mid z), p(h \mid q)$ and $p(q)$ are given in the appendix. Then, the posterior $\log$-likelihood of $\mathbf{z}$ can then be written as [16]

$$
\begin{aligned}
& L(\mathbf{z} \mid \mathbf{x})=-\left(\mathbf{x}-\mathbf{S}_{\lambda} \mathbf{h}\right)^{H} \mathbf{A}_{n}\left(\mathbf{x}-\mathbf{S}_{\lambda} \mathbf{h}\right)-\frac{\mathbf{h}^{H} \mathbf{D}_{\mathbf{q}} \mathbf{h}}{2 \sigma_{1}^{2}} \\
& -\frac{\mathbf{h}^{H}\left(1-\mathbf{D}_{\mathbf{q}}\right) \mathbf{h}}{2 \sigma_{0}^{2}}+\mathbf{q}^{H} \mathbf{q} \ln \left(\frac{\mu}{1-\mu} \frac{\sigma_{0}^{2}}{\sigma_{1}^{2}}\right)+C
\end{aligned}
$$

where $\mathbf{D}_{\mathbf{q}}$ represents the diagonal matrix with the k-th entry equal to $q_{k}$ and $C$ is a constant term and $\mathbf{A}_{n}$ is the inverse of noise covariance matrix.

\section{B. MCMC algorithm}

Usually, in the bayesian framework, one wants to find the value of the parameter vector that maximizes the posterior likelihood. This approach is known as the Maximum A Posteriori (MAP) approach. Here, the MAP estimator of $\mathbf{z}=(\mathbf{q}, \mathbf{h})$ is given by

$$
(\hat{\mathbf{q}}, \hat{\mathbf{h}})=\arg \max _{(\mathbf{q}, \mathbf{h})} L(\mathbf{z} \mid \mathbf{x})
$$

Therefore, following the MAP approach would lead to find the global maximum of $L(\mathbf{z} \mid \mathbf{x})$ over all possible sequences $(\mathbf{q}, \mathbf{h})$. This is a very complex combinatorial problem, since there are $2^{N}$ possible sequences $\mathbf{q}$. Several methods have been proposed to overcome this problem by iteratively improving the maximisation. This is the case for instance of the SMLR algorithm [9] and its variations [3]. However, due to their deterministic nature, these algorithms are dependent to initial conditions and may converge towards a local optimum. Another possibility is to use the well-known Simulated Annealing (SA) method [10] to find the global optimum of the problem. However this method converges very slowly to the solution, especially when the parameter space is large, which is the case here. If the MAP estimator is therefore difficult to reach, it is however possible to resort to another method, based on MCMC trials, that have proved to yield very good results with a reasonable computational cost. The idea is to generate realizations of a given process and then use these realizations to compute an empirical estimator of the parameters. Thus, the algorithm aims to simulate the marginal posterior pdf $p(\mathbf{z} \mid \mathbf{x})$ associated with Eq. (19). This is carried out by a Gibbs sampler that simulates realizations of samples $z_{k}$ according to the a posteriori marginals $p\left(z_{k} \mid x, z_{-k}\right)$, where $z_{k}=\left(z_{0}, \cdots, z_{k-1}, z_{k+1}, \ldots, z_{L}\right)$.

Implementation of the Gibbs sampler is supplied in the appendix.

Let $\left(z_{i=1, I}^{(i)}\right)$ denote the simulated vectors supplied by operating the Gibbs sampler I times on the L entries of $z$. After a certain learning period corresponding to $I_{0}$ iterations, the Gibbs sampler reaches 'thermal equilibrium', that is, the generated samples are distributed according to the a posteriori probability $p(\mathbf{z} \mid \mathbf{x})$.

For our tests, we chose $I_{0}=100$ and $I=500$. The samples are then used to compute the following estimators $\hat{\mathbf{q}}$ and $\hat{\mathbf{h}}$ of $\mathbf{q}$ and $\mathbf{h}$ :

$$
\begin{aligned}
& \hat{q}_{k}=\left\{\begin{array}{rrr}
1 & \text { if } & \frac{1}{I-I_{0}} \sum_{i=I_{0}+1}^{I} q_{k}^{(i)}>s \\
0 & & \text { otherwise }
\end{array}\right. \\
& \hat{h}_{k}=\left\{\begin{array}{llc}
\frac{\sum_{i=I_{0}+1}^{I} q_{k}^{(i)} h_{k}^{(i)}}{\sum_{i=I_{0}+1}^{I} q_{k}^{(i)}} & \text { if } & q_{k}=1 \\
0 & & \text { otherwise. }
\end{array}\right.
\end{aligned}
$$

where the threshold $s$ was set to 0.5 .

This choice is appropriate when no preference is made 
between the two kinds of error that may arise, that is deciding $\hat{q}_{k}=1$ when $q_{k}=0$ and deciding $\hat{q}_{k}=0$ when $q_{k}=1$. It can indeed be proved that the value $s=0.5$ minimizes the Bayes risk when uniform equal costs are chosen.

\section{Simulations AND RESUlTS}

We carried out tests on BOCsin( 1,1$)$ modulated signals.

\section{A. Fixed parameters}

The window size on which we achieved the search for the delay is four chips, that is, twice the auto correlation peak size. To keep coherence among simulation experiments, for all the methods we set to the same value the sampling frequency $f_{s}=120 \mathrm{MHz}$, the low-pass front-end filter band $B=2.5 \mathrm{MHz}$, the $\mathrm{SNR}-20 \mathrm{~dB}$ and the number of simulations $N=1000$.

\section{B. Results}

We consider the propagation through a simulated channel according to the model described in Figure 5. We carried out 1000 simulations with distinct randomly chosen channels. We assume that good detection is achieved when the first path position is close to the true line of sight path within a range of $\pm \frac{1}{3} T_{c}$ for a BOCsin(1,1) signal and $T_{c}$ for a GPS C/A signal.

The Figure 11 represents the RMSE (Root Mean Square Error) of the first detected path delay compared to the time pseudo distance for the model described in Fig 5. We have chosen $\mu=2$ for the Poisson distribution of the number of paths, leading to an average number of paths equal to 3 for each simulations. The Figure 11 clearly shows that the MCMC method estimates better the time delay for both a BOCsin(1,1) modulated signal and a GPS C/A modulated signal than the classical approach. These results were obtained for a channel where the path separations are lower than 1 chip duration which is why, as we can notice here, the BOCsin $(1,1)$ is not well estimated by the classical approach because of the ambiguity between the main peak and the side peaks of the autocorrelation function. However, the MCMC method distinguishes well between the peaks and gives a much lower square error.

Now, we consider another channel derived by Steingass. In Figure 12, because the path separations are wider, the BOCsin $(1,1)$ is better estimated by the classical approach than the GPS C/A signal. In this channel configuration, the MCMC method still provides better results.

Similarly, we have checked that the algorithm brings improvement compared to the standard approach when applied to AltBOC $(15,10)$ signaling.

Now, let us consider the consequences of these results in a scenario positioning. Here, the performance of simulated positioning is given in the following table. The user is in

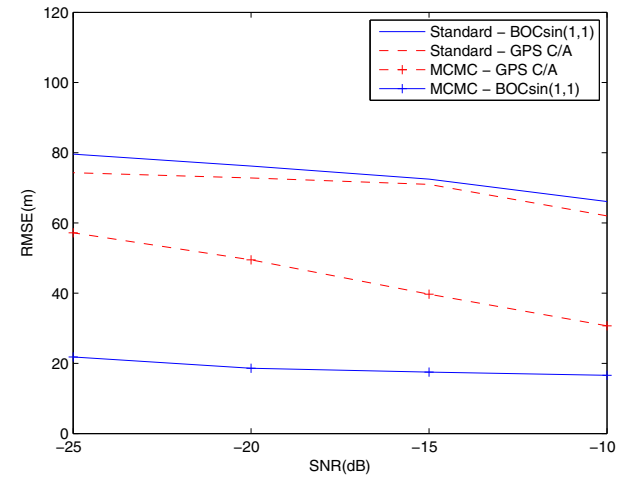

Fig. 11. RMSE due to simulated multipath channel for model of Figure 5

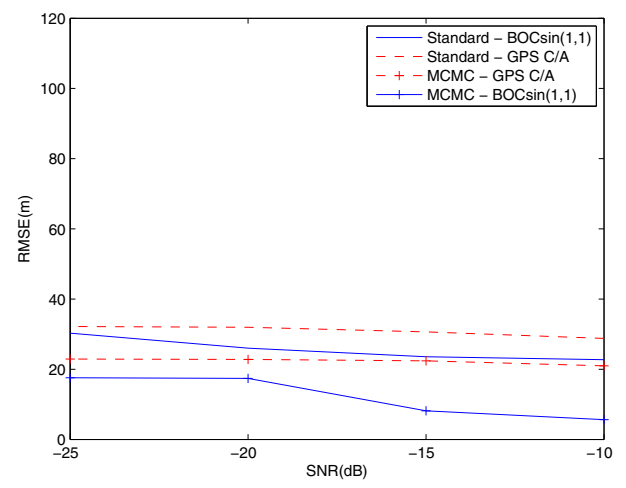

Fig. 12. RMSE due to Steingass multipath channel for Steingass model

Monterey on 05/04/2008 at 3:30 PM, the Galileo satellites in view are shown in Figure 13, and we consider all the satellites that have an elevation $>20$. A least square method is used for triangulation based on estimated delays with performance similar to that obtained in table II. To have these results, we have added noise estimation on pseudo-distances according to model in Fig. 11. Noise variances are chosen depending on satellite elevations according to table I.

TABLE I

SATELLITE ELEVATIONS AND CORRESPONDING SNR TAKEN FOR SIMULATIONS OF FIG. 13 AND 14

\begin{tabular}{|c|c|}
\hline Satellite elevation & SNR $(\mathrm{dB})$ \\
\hline$[20,40[$ & -25 \\
\hline$[40,60[$ & -20 \\
\hline$[60,80[$ & -15 \\
\hline$[80,90]$ & -10 \\
\hline
\end{tabular}


TABLE II

POSITIONING ERRORS FOR BOCSIN $(1,1)$ SIGNAL

\begin{tabular}{|c|c|c|c|c|}
\hline RMSE & $\mathrm{x}$ & $\mathrm{y}$ & $\mathrm{z}$ & std \\
\hline MCMC $(\mathrm{m})$ & 27.1 & 37.2 & 41.9 & 35.4 \\
\hline Standard $(\mathrm{m})$ & 103.8 & 140.3 & 168.2 & 137.4 \\
\hline
\end{tabular}

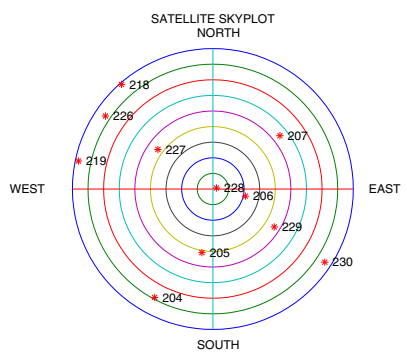

Fig. 13. Satellites in view

We do the same scenario with the GPS constellation, and we gain 30 meters accuracy on average.

TABLE III

POSITIONING ERRORS FOR GPS C/A SIGNAL

\begin{tabular}{|c|c|c|c|c|}
\hline RMSE & $\mathrm{x}$ & $\mathrm{y}$ & $\mathrm{z}$ & $\mathrm{std}$ \\
\hline MCMC $(\mathrm{m})$ & 59.6 & 71.8 & 79.6 & 70.3 \\
\hline Standard $(\mathrm{m})$ & 83.8 & 103.8 & 115.5 & 101.0 \\
\hline
\end{tabular}

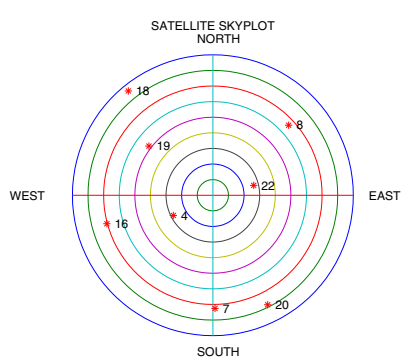

Fig. 14. Satellites in view

\section{CONCLUSION}

In this paper, we have shown that using the classical acquisition method to determine an interesting reduced search zone, and then looking for the code delay by means of the MCMC algorithm in this zone, the multipath effect is clearly reduced. In fact, we have developed a high performance acquisition system thanks to the MCMC algorithm, namely in terms of accuracy. In addition, the reduced search zone enables the MCMC method to converge in a short time. Obviously, the MCMC method represents a good trade-off between estimation accuracy and computational complexity.

\section{APPENDIX}

\section{A. Posterior distribution}

From Bayes formula,

$$
p(\mathbf{z} \mid \mathbf{x}) \propto p(\mathbf{x} \mid \mathbf{z}) p(\mathbf{z})=p(\mathbf{x} \mid \mathbf{z}) p(\mathbf{h} \mid \mathbf{q}) p(\mathbf{q})
$$

assuming that

1) the noise is complex circular Gaussian with autocorrelation matrix $\Gamma_{n}=A_{n}^{-1}$

2) samples $q_{k}$ are independent

3) samples $h_{k}$ are independent conditionally to $q_{k}$, there results

$$
p(\mathbf{x} \mid \mathbf{z})=\frac{\left|\mathbf{A}_{n}\right|}{\pi^{L}} \exp \left(-\left(\mathbf{x}-\mathbf{S}_{\Lambda} \mathbf{h}\right)^{H} \mathbf{A}_{n}\left(\mathbf{x}-\mathbf{S}_{\Lambda} \mathbf{h}\right)\right)
$$

$$
p(\mathbf{h} \mid \mathbf{q})=\prod_{k=1}^{L} p\left(h_{k} \mid q_{k}\right)
$$

$p\left(h_{k} \mid q_{k}\right)=\left[\frac{\exp \left(-\frac{\left|h_{k}\right|^{2}}{2 \sigma_{1}^{2}}\right)}{2 \pi \sigma_{1}^{2}}\right]^{q_{k}}\left[\frac{\exp \left(-\frac{\left|h_{k}\right|^{2}}{2 \sigma_{0}^{2}}\right)}{2 \pi \sigma_{0}^{2}}\right]^{1-q_{k}}$

$$
p(\mathbf{q})=\prod_{k=1}^{L} p\left(q_{k}\right)=\prod_{k=1}^{L} \mu^{q_{k}}(1-\mu)^{1-q_{k}}
$$

\section{B. Gibbs sampling}

The Gibbs sampler is implemented in the following way:

1) Initialization: $\mathbf{q}=\mathbf{q}^{0}$ and $\mathbf{h}=\mathbf{h}^{0}$

2) For $i \geq I$ and for $k$ randomly covering $\{1, \cdots, L\}$,

a) Detection step:

i) calculate $d_{k}=p\left(q_{k}=1 \mid \mathbf{x}, \mathbf{z}_{-k}^{(i)}\right)$

ii) $\operatorname{draw} q_{k}^{(i)}=\mathbb{1}_{d_{k} \geq v}$, with $v \sim U_{[0,1]}$

b) Estimation step:

i) draw

$$
h_{k}^{(i)} \sim N\left(m_{q_{k}^{(i)}}, V_{q_{k}^{(i)}}\right)+j N\left(m_{q_{k}^{(i)}}, V_{q_{k}^{(i)}}\right)
$$

where $U_{A}$ is the uniform distribution on $A$,

$$
\mathbb{1}_{A}(t)= \begin{cases}1 & \text { if } t \in A \\ 0 & \text { otherwise }\end{cases}
$$

$$
m_{i}=2 V_{i} \sum_{n=0}^{M} \sum_{p=0}^{M} s_{p}^{*} a_{n+k, p+k} U_{k, n}
$$

$$
V_{i}=\left(\frac{1}{\sigma_{i}^{2}}+2 \sum_{p=0}^{M} s_{n}^{*} a_{n+k, p+k} s_{p}\right)^{-1}
$$

$$
d_{k}=\left[1+\frac{1-\mu}{\mu} \frac{V_{0}}{V_{1}} \frac{\sigma_{1}^{2}}{\sigma_{0}^{2}} \exp \left(\frac{\left|m_{0}\right|^{2}}{2 V_{0}}-\frac{\left|m_{0}\right|^{2}}{2 V_{0}}\right)\right]^{-1} .
$$




\section{REFERENCES}

[1] J. W. Betz. Design and Performance of Code Tracking for the GPS M Code Signal. In Proceedings of the 13th International Technical Meeting of the Satellite Division of the Institute of Navigation ION GPS 2001, pages $2140-2150$, September 2000.

[2] S. Bourguignon and H. Carfantan. Bernoulli-Gaussian Spectral Analysis of Unevenly Spaced Astrophysical Data. In IEEE Workshop on Statistical Signal Processing, pages 811-816, July 2005.

[3] F. Champagnat, Y. Goussard, and J. Idier. Unsupervised deconvolution of sparse spike trains using stochastic approximation. IEEE Trans. Signal Processing, 44:2988-2998, 1996.

[4] S.F. Cotter and B.D. Rao. Sparse Channel Estimation via Matching Poursuit With Application to Equalisation. IEEE Transactions on communications, 50(3):374-377, March 2002.

[5] M. Feder and E. Weinstein. Parameter Estimation Of Superimposed Signals Using the EM Algorithm. IEEE Transactions on acoustics, speech and signal processing, 36(4):477-489, April 1988.

[6] S. Gligorevic. Joint Channel Estimation And Equalization For Fast Time-Variant Multipath Channels. In IEEE PIMRC, 2002.

[7] H. Hashemi. The Indoor Radio Propagation Channel. In Proceedings of IEEE, volume 81, pages 943-968, July 1993.

[8] E. Kaplan and C. Hegarty. Understanding GPS: Principles And Applications. Artech House Publishers, 2 edition, December 2005.

[9] J. Kormylo and J. Mendel. Maximum-likelihood Detection and Estimation of Bernoulli-Gaussian Processes. IEEE Transactions on Information Theory, 28(3):482-488, May 1982.

[10] P. V. Laarhoven and E. Aarts. Simulated annealing: theory and applications. D. Reidel Publishing Company, 1987.

[11] G. Lachapelle, H. Kuunsniemi, D.T.H. Dao, G. MacGougan, and M.E. Cannon. HSGSP Signal Analysis and Performance under various Indoor
Conditions. In ION GPS/GNSS conference, Portland, Oregon, September 2003.

[12] M. Lavielle. Bayesian Deconvolution of Bernoulli-Gaussian Processes. Signal processing, 33(1):67-79, July 1993

[13] H. K. Lee, J.G. Lee, and G.I. Jee. GPS multipath detection based on sequence of successive-time double-differences. Signal Processing Lettersn IEEE, 11(3):316- 319, March 2004.

[14] T. Lo, J. Litva, and H. Leung. A New Approach for Estimating Indoor Radio Propagation Characteristics. IEEE Transactions on Antennas and Propagation, 42(10):1369-1376, October 1994.

[15] F. Perez-Fontan, B. Sanmartin, A. Steingass ans A. Lehner, J. Selva, E. Kubista, and B. Arbesser-Rastburg. Measurements and Modelling of the Satellite-to-Indoor Channel for Galileo. In IEEE PLANS, Monterey, California USA, April 2004.

[16] O. Rabaste and T. Chonavel. Estimation of multipath channels with long impulse response at low SNR, via an MCMC method. IEEE Transactions on Signal Processing, 55(4):1312-1325, April 2007.

[17] S. Rappaport. Wireless Communications: Principles and Practice. IEEE Press, 1996.

[18] E. Rebeyrol, C. Macabiau, L. Lestarquit, L. Ries, J.L Issler, M.L. Boucheret, and M. Bousquet. BOC Power Spectrum Densities. In ION NTM, pages 769-778, San Diego CA, 24-26 Janvier 2005.

[19] O. Rosec and J.M. Boucher. A Bayesian Method for Ocean Acoustic Tomography in the presence of transducer distorsion. In Fifth European Conference on Underwater Acoustics, Lyon, France, July 2000.

[20] A. Steingass and A. Lehner. Measurement of the Navigation Multipath Channel : A Statistical Analysis. In ION GNSS 17th International Technical Meeting of the Satellite Division, Long Beach, California, September 2004.

[21] A. Steingass and A. Lehner. A Channel Model for Land Mobile Satellite Navigation. In GNSS Conference, Munich, Germany, July 2005. 